\title{
Prebiotic galacto-oligosaccharides mitigate the adverse effects of iron fortification on the gut microbiome: a randomised controlled study in Kenyan infants
}

\author{
Daniela Paganini, ${ }^{1}$ Mary A Uyoga, ${ }^{2}$ Guus A M Kortman, ${ }^{3}$ Colin I Cercamondi, ${ }^{1}$ \\ Diego Moretti, ${ }_{1}^{1}$ Tanja Barth-Jaeggi, ${ }^{4}$ Clarissa Schwab, ${ }^{1}$ Jos Boekhorst, ${ }^{3}$ \\ Harro M Timmerman, ${ }^{3}$ Christophe Lacroix, ${ }^{1}$ Simon Karanja, ${ }^{2}$ Michael B Zimmermann ${ }^{1}$
}

\begin{abstract}
- Additional material is published online only. To view please visit the journal online (http://dx.doi.org/10.1136/ gutjnl-2017-314418).

${ }^{1}$ Department of Health Sciences and Technology, ETH Zurich,

Zurich, Switzerland

${ }^{2}$ Department of Medical Epidemiology, College of Health Sciences, Jomo Kenyatta University of Agriculture and Technology, Nairobi, Kenya ${ }^{3}$ NIZO Food Research BV, Ede, The Netherlands

${ }^{4}$ Health Systems Support Unit Swiss Tropical and Public Health Institute, Basel, Switzerland
\end{abstract}

Correspondence to Professor Michael B Zimmermann, ETH Zurich, LFVD20, CH\#8092, Zurich, Switzerland; michael. zimmermann@hest.ethz.ch

Received 1 May 2017 Revised 30 May 2017 Accepted 30 May 2017 Published Online First 3 August 2017

\section{SLinked}

- http://dx.doi.org/10.1136/ gutjnl-2017-314593

CrossMark

To cite: Paganini $D$,

Uyoga MA, Kortman GAM

et al. Gut

2017:66:1956-1967.

\begin{abstract}
Objective Iron-containing micronutrient powders

(MNPs) reduce anaemia in African infants, but the current high iron dose $(12.5 \mathrm{mg} /$ day) may decrease gut Bifidobacteriaceae and Lactobacillaceae, and increase enteropathogens, diarrhoea and respiratory tract infections (RTIS). We evaluated the efficacy and safety of a new MNP formula with prebiotic galactooligosaccharides (GOS) combined with a low dose $(5 \mathrm{mg} /$ day) of highly bioavailable iron.
\end{abstract}

Design In a 4-month, controlled, double-blind trial, we randomised Kenyan infants aged 6.5-9.5 months $(n=155)$ to receive daily (1) a MNP without iron (control); (2) the identical MNP but with $5 \mathrm{mg}$ iron $(2.5 \mathrm{mg}$ as sodium iron ethylenediaminetetraacetate and $2.5 \mathrm{mg}$ as ferrous fumarate) (Fe group); or (3) the identical MNP as the Fe group but with $7.5 \mathrm{~g} \mathrm{GOS} \mathrm{(FeGOS} \mathrm{group).}$

Results Anaemia decreased by $\approx 50 \%$ in the Fe and FeGOS groups $(p<0.001)$. Compared with the control or FeGOS group, in the Fe group there were (1) lower abundances of Bifidobacterium and Lactobacillus and higher abundances of Clostridiales $(p<0.01)$; (2) higher abundances of virulence and toxin genes (VTGs) of pathogens $(p<0.01)$; $(3)$ higher plasma intestinal fatty acid-binding protein (a biomarker of enterocyte damage) $(p<0.05)$; and (4) a higher incidence of treated RTIs ( $p<0.05)$. In contrast, there were no significant differences in these variables comparing the control and FeGOS groups, with the exception that the abundance of VTGs of all pathogens was significantly lower in the FeGOS group compared with the control and Fe groups $(p<0.01)$.

Conclusion A MNP containing a low dose of highly bioavailable iron reduces anaemia, and the addition of GOS mitigates most of the adverse effects of iron on the gut microbiome and morbidity in African infants.

Trial registration number NCT02118402.

\section{INTRODUCTION}

The WHO estimates that $62.3 \%$ of preschool children in Africa are anaemic, ${ }^{1}$ of which $\approx 50 \%$ is attributable to iron deficiency anaemia (IDA). ${ }^{2}$ Iron-containing micronutrient powders (MNPs) added to complementary foods effectively reduce IDA in infants, a strategy described as in-home

\section{Significance of this study}

What is already known on this subject?

- Iron-containing micronutrient powders (MNPs) are widely used in Sub-Saharan Africa to reduce anaemia.

- Current MNPs contain a high daily iron dose (12.5 mg/day) that may decrease gut Bifidobacteriaceae and Lactobacillaceae, and increase enteropathogens, diarrhoea and respiratory tract infections (RTIs); thus, safer MNP formulations are needed.

- Prebiotic galacto-oligosaccharides (GOS), commonly added to infant formula, can selectively enhance growth of Bifidobacteriaceae and Lactobacillaceae.

What are the new findings?

- A new MNP formulation containing a low dose ( $5 \mathrm{mg} /$ day) of highly bioavailable iron reduces anaemia in Kenyan infants.

- The addition of $7.5 \mathrm{~g} \mathrm{GOS}$ to this low-iron MNP mitigates most of the adverse effects of iron on the gut microbiome, resulting in greater abundances of Bifidobacterium and Lactobacillus, lower abundances of virulence and toxin genes of pathogens, less enterocyte damage, and a lower incidence of treated RTIs.

How might it impact on clinical practice in the foreseeable future?

- Our findings demonstrate that the iron dose in MNPs can be reduced to $5 \mathrm{mg}$ while maintaining efficacy, and that prebiotics given with low-iron MNPs in the African setting may be beneficial to reduce the adverse effects of iron on the infant gut.

fortification'. ${ }^{3}$ However, current MNPs contain a high iron dose $(12.5 \mathrm{mg})$ that is equivalent to the dose provided by oral iron supplements for the treatment of IDA in infancy. ${ }^{3}$ The safety of these MNPs is uncertain, ${ }^{45}$ as recent MNP trials $(12.5 \mathrm{mg}$ iron/day) in infants reported increases in diarrhoea and respiratory tract infections (RTIs). ${ }^{6}{ }^{7}$ Iron 
absorption from MNPs by African infants is only $4 \%-9 \%{ }^{8}$; thus, most of the iron passes unabsorbed into the infant colon. Iron is a growth-limiting nutrient for many gut bacteria, ${ }^{9}$ and for most enteric Gram-negative bacteria (eg, Salmonella, pathogenic Escherichia coli) iron acquisition is essential for virulence and colonisation. ${ }^{10}$ In contrast, lactobacilli and bifidobacteria can improve gut integrity and reduce colonisation by enteric pathogens, ${ }^{11}{ }^{12}$ but lactobacilli require no iron ${ }^{13}$ and some Bifidobacterium spp can efficiently sequester iron, limiting its availability to pathogens. ${ }^{14}$ Large increases in colonic iron could favour pathogen growth, especially in African infants in areas of poor hygiene who have high carriage rates of pathogens. ${ }^{15}$ In a trial in Kenyan infants, MNPs increased the Enterobacteriaceae:Bifidobacteriaceae ratio, numbers of enteropathogenic E. coli, gut inflammation and diarrhoea. ${ }^{15}$

Thus, safer MNP formulations are needed. One strategy could be to reduce the iron dose but at the same time maximise absorption by providing iron as sodium iron ethylenediaminetetraacetate (NaFeEDTA), a chelated highly bioavailable iron compound, along with a phytase to reduce phytic acid in complementary foods, an inhibitor of iron absorption. ${ }^{16}$ A second strategy could be to include a component that potentially mitigates the adverse effects of fortification iron on the gut microbiome, such as a prebiotic, that could maintain and/or promote growth of the beneficial bacteria in the infant gut. ${ }^{12} 17$ Galacto-oligosaccharides (GOS) selectively enhance growth of Bifidobacteriaceae and Lactobacillaceae, and increase colonic production of short-chain fatty acids (SCFAs) that decrease luminal $\mathrm{pH}$, which may reduce growth of enteropathogens. ${ }^{12} 17$ GOS has Generally Recognized as Safe (GRAS) status ${ }^{18}$ and is often added to commercial infant formula. $^{12}$

We hypothesised that (1) a MNP formula containing $5 \mathrm{mg}$ of highly bioavailable iron would reduce anaemia; and (2) the addition of GOS to a MNP containing $5 \mathrm{mg}$ of iron would mitigate the adverse effects of iron on the infant gut microbiome and inflammation. Coprimary outcomes were (1) the gut microbiome assessed by $16 \mathrm{~S}$ rDNA sequencing (the taxa of primary interest: Bifidobacterium, Lactobacillus, Enterobacteriaceae, Clostridiales and Bacteroidetes); (2) enteropathogen abundance assessed by quantitative PCR (qPCR) of virulence and toxin genes (VTGs); and (3) gut inflammation/integrity assessed by faecal calprotectin and plasma intestinal fatty acid-binding protein (I-FABP). Secondary outcomes were haemoglobin $(\mathrm{Hb})$, iron status and morbidity.

\section{MATERIALS AND METHODS Study design}

We conducted the study from October 2014 to December 2015 in southern coastal Kenya. Based on previous studies in African infants and children, ${ }^{15} 19$ we estimated a sample size of $\approx 35$ infants in each group would be sufficient to detect a difference of approximately $0.85 \mathrm{log}$ number of copies/g faeces in Lactobacillaceae and Bifidobacteriaceae, considering an SD of 1.25 in $\log$ abundance, with $\alpha=0.05$ and $\beta=0.8$. We anticipated an attrition rate of $\approx 30 \%$ and planned to enrol 50 infants per group. The study was approved by the ethics and research committees of the Kenyatta National Hospital/University of Nairobi, Kenya (P521/10/2013) and the Zurich Cantonal Ethical Commission (2014-0232). Caregivers gave informed consent by either a written signature or a fingerprint. An independent Data Safety Monitoring Board (DSMB) monitored the study.

We prescreened 334 infants (study design, figure 1) for the following inclusion criteria: (1) age 6.5-9.5 months; (2) no chronic or acute illnesses; (3) no vitamin and mineral supplements in the previous 8 weeks; (4) no antibiotic treatment in the previous 10 weeks; and (5) anticipated residence in the area for the study duration. For 199 infants, we received informed consent and we enrolled them into a 2 -week run-in period without MNPs to familiarise the infants and caregivers with preparation and consumption of the maize porridge; 171 infants completed the 2 -week run-in period and were invited for the complete baseline screening. Inclusion criteria during the baseline screening were (1) $\mathrm{Hb}>70 \mathrm{~g} / \mathrm{L}$; and (2) Z scores for weight-for-age and weight-for-height both $>-3$. Finally, 155 infants met the inclusion criteria and were randomly assigned in consecutive order to the three intervention groups using a computer-generated list and three colour codes. The first (control group) received daily a MNP sachet containing $30 \mathrm{mg}$ of ascorbic acid and other vitamins and minerals, 190 phytase unit (FTU) of a food-grade microbial phytase (Tolerase 20000G, DSM Nutritional Products, Kaiseraugst, Switzerland), ${ }^{16}$ and as filler, $10.5 \mathrm{~g}$ of maltodextrin. The second (Fe group) received the identical MNP but with $2.5 \mathrm{mg}$ iron as NaFeEDTA and $2.5 \mathrm{mg}$ iron as ferrous fumarate. The third (FeGOS group) received the identical MNP as the second group, except the maltodextrin was replaced with $10.5 \mathrm{~g}$ of $75 \%$ GOS (Vivinal GOS 75 Powder, FrieslandCampina, Wageningen, The Netherlands). GOS consists of chains of galactose units of varying length: the degree of polymerisation (DP) of the GOS used in this study (on weight percentage of oligosaccharide) was 31\% DP2 (other than lactose), 38\% DP3, 18\% DP4, 8\% DP5 and 5\% DP6 and higher (FrieslandCampina). The composition of the MNPs is shown in table 1.

The MNPs were packed in colour-coded sachets containing a daily dose, and the codes were kept by the DSMB; study participants and investigators were masked to group assignment. A baseline faecal sample and a venous blood sample were collected from the infants before the MNPs were distributed. Caregivers were carefully instructed on faecal sample collection and were given plastic diapers, spatulas and screw-cap plastic containers containing a carbon dioxide generator system to create an anaerobic atmosphere (Microbiology Anaerocult A mini, Merck, Darmstadt, Germany). The faecal samples were collected at home into the containers, and the study team aliquoted and stored them at $-20^{\circ} \mathrm{C}$ the same day until further analysis. We measured infant weight and height, and recorded demographic characteristics, brief medical history and feeding habits of the infants using a questionnaire and local health records.

Weekly for 4 months, we dispensed seven MNP sachets and $2 \mathrm{~kg}$ of unfortified, refined maize flour to the families. During weekly visits, we assessed compliance by questioning the caregiver and collecting the previous week's used and unused MNP sachets. At the weekly visits, we assessed infant morbidity over the previous week as described in the online supplementary material. After 3 weeks and 4 months of the intervention, we collected a faecal sample, and at 4 months we collected a venous blood sample and measured infant weight and length as described above. Any infants remaining anaemic at 4 months were examined and treated by the study clinicians according to local guidelines.

\section{Laboratory methods \\ Blood samples}

At baseline and 4 months, we collected a $3 \mathrm{~mL}$ venous blood sample. We measured $\mathrm{Hb}$ on the day of collection by using a HemoCue 300 analyser (HemoCue, Angelholm, Sweden) and separated plasma by centrifugation and froze it on the day of 


\section{Invited for pre-screening: $\mathrm{n}=334$ infants}

Excluded ( $n=135)$

- Antibiotic use past 10 weeks $(n=44)$

- Consent withdrawal $(n=56)$

- Relocation ( $n=27)$

- Consumption of iron supplements $(n=4)$

- Other health condition $(n=2)$

- Severe underweight $(n=1)$

- Date of birth unverifiable $(n=1)$

Included in the 2-week run-in period: $n=199$ infants

Excluded during the 2-week run-in period or at baseline $(n=44)$

- Antibiotic use $(n=19)$

- Consent withdrawal $(n=9)$

- Failed venipuncture $(n=7)$

- Severe anaemia $(n=5)$

- Severe underweight $(n=4)$

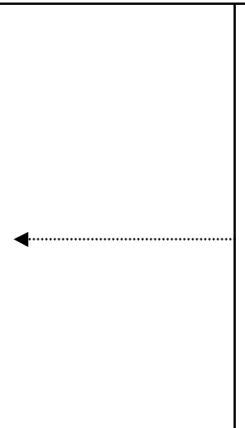

Figure 1 Study design and participants flow chart. FeFum, ferrous fumarate; GOS, galacto-oligosaccharides; MNP, micronutrient powder; NaFeEDTA, sodium iron ethylenediaminetetraacetate. 


\begin{tabular}{ll}
\hline $\begin{array}{l}\text { Table } 1 \text { Composition of the three MNP formulations used in the } \\
\text { study }\end{array}$ & Amount per sachet \\
\hline Components & \\
\hline All groups & $400 \mu \mathrm{g}$ \\
\hline Vitamin A & $5 \mu \mathrm{g}$ \\
\hline Vitamin D & $5 \mathrm{mg}$ \\
\hline Tocopherolequivalents & $0.5 \mathrm{mg}$ \\
\hline Thiamine & $0.5 \mathrm{mg}$ \\
\hline Riboflavin & $0.5 \mathrm{mg}$ \\
\hline Vitamin B & $90 \mu \mathrm{g}$ \\
\hline Folic acid & $6 \mathrm{mg}$ \\
\hline Niacin & $0.9 \mu \mathrm{g}$ \\
\hline Vitamin $\mathrm{B}_{12}$ & $30 \mathrm{mg}$ \\
\hline Vitamin C & $0.56 \mathrm{mg}$ \\
\hline Copper & $90 \mu \mathrm{gg}$ \\
\hline lodine & $17 \mu \mathrm{g}$ \\
\hline Selenium & $4.1 \mathrm{mg}$ \\
\hline Zinc & $190 \mathrm{FTU}$ \\
\hline Phytase & \\
\hline Control group & Add to $11 \mathrm{~g}$ \\
\hline Maltodextrin & $2.5 \mathrm{mg}$ \\
\hline Fe group & $2.5 \mathrm{mg}$ \\
\hline Iron (as ferrous fumarate) & Add to $11 \mathrm{~g}$ \\
\hline Iron (as NaFeEDTA) & $2.5 \mathrm{mg}$ \\
\hline Maltodextrin & $2.5 \mathrm{mg}$ \\
\hline FeGOS group & $7.5 \mathrm{~g}$ as $10.5 \mathrm{~g} \mathrm{GOS} \mathrm{-75}$ \\
\hline Iron (as ferrous fumarate) & \\
\hline Iron (as NaFeEDTA) & \\
\hline Galacto-oligosaccharides (GOS) & \\
\hline
\end{tabular}

FTU, phytase unit; MNP, micronutrient powder; NaFeEDTA, sodium iron ethylenediaminetetraacetate.

collection until analysis. Plasma ferritin (PF), soluble transferrin receptor (sTfR), C reactive protein and alpha-glycoprotein were analysed using a multiplex immunoassay, ${ }^{20}$ and I-FABP by using a commercial available ELISA (Hycult Biotech, Uden, The Netherlands). Reference values are given in the online supplementary material.

\section{Faecal samples}

We measured faecal calprotectin using the Calprest ELISA assay for stools, following the manufacturer's procedures (Eurospital, Trieste, Italy) and faecal $\mathrm{pH}$. Details of the method and reference values are given in the online supplementary material. We extracted and quantified faecal DNA as described in the online supplementary material. Using $\mathrm{qPCR}$, we targeted VTGs of selected enteropathogenic bacteria: Bacillus cereus, Clostridium difficile, Clostridium perfringens, enterohemorrhagic E.coli (EHEC), enteropathogenic E.coli (EPEC) and enterotoxigenic E.coli (ETEC), Salmonella spp, and Staphylococcus aureus; details and primers are given in online supplementary table 1 . These pathogens were chosen based on their common occurrence in Kenyan infants in our previous study. ${ }^{15}$ Barcoded amplicons from the V3-V4 region of $16 \mathrm{~S}$ rRNA genes were generated using a two-step PCR, and universal primers appended with Illumina adapters were used for initial amplification of the V3-V4 part of the 16S rRNA gene. PCR products were purified and quantified, followed by multiplexing, clustering and sequencing on an Illumina MiSeq with the paired-end (2x) $300 \mathrm{bp}$ protocol and indexing; details are given in the online supplementary material.

\section{Statistical methods}

We analysed biochemical and anthropometric data using the $\mathrm{R}$ statistical programming environment (R V.3.2.3, 2015). Normally distributed data were expressed as means and SD; non-normally distributed data as median and ranges or IQRs. When data were non-normally distributed, appropriate transformation of values was performed before statistical analysis. Details of the methods of comparison for the haematological, biochemical and microbiological data are given in the online supplementary material. Because we felt near significant $p$ values should not be routinely ignored in this study, as some of these may provide interesting hints on the relationships of iron and GOS with the complex and still poorly understood infant gut microbiome, we mention $\mathrm{p}$ values $<0.1$ as statistical trends. We considered $\mathrm{p}$ values $<0.05$ as statistically significant.

\section{RESULTS}

During the intervention, 10 infants dropped out (figure 1), and the final analysis was completed on 145 infants: control group $(n=48)$, Fe group $(n=49)$ and FeGOS group $(n=48)$. At baseline, all infants were still partially breast fed except one infant in the Fe group, and all were already receiving complementary foods, mainly maize porridge; $97 \%$ of infants were still partially breast fed at endpoint. The mean \pm SD native iron concentration in the maize flour used for porridge preparation was $1.02 \pm 0.13 \mathrm{mg} / 100 \mathrm{~g}$ dry matter. Compliance with the MNP sachets in the control, Fe and FeGOS groups was 94\%, 95\% and $94 \%$, respectively.

\section{Haematological and inflammation status}

Table 2 shows, at baseline and 4 months, age, gender, iron and inflammation status. At baseline, there were no significant group differences. At 4 months, there were no significant group differences in any of the inflammation variables. At 4 months, compared with the control group, $\mathrm{Hb}$ and $\mathrm{PF}$ were higher in the Fe and FeGOS groups ( $\mathrm{p}<0.001$ for both); sTfR was lower in the Fe and FeGOS groups $(\mathrm{p}<0.001$ for both); and the prevalence of anaemia and IDA was lower in the Fe and FeGOS group ( $\mathrm{p}<0.001$ for both). There were no significant differences between $\mathrm{Fe}$ and FeGOS at 4 months in any of these haematological variables. Online supplementary table 2 shows there were no significant differences in anthropometric variables between groups during the study.

\section{Gut microbiome by $16 \mathrm{~S}$ rDNA sequencing}

On average 23902 bacterial $16 \mathrm{~S}$ rDNA sequences per sample were analysed by $16 \mathrm{~S}$ profiling. At baseline, the microbiome consisted of the phyla Actinobacteria $(66.4 \%$ of the $16 \mathrm{~S}$ rDNA reads), which was mostly represented by the family Bifidobacteriaceae, contributing $60.5 \%$ to the total $16 \mathrm{~S}$ rDNA, Firmicutes (28.6\%; including 4.6\% Lactobacillus), Bacteroidetes (2.1\%; including 0.6\% Bacteroides and 1.3\% Prevotella) and Proteobacteria (2.6\%), with the predominant family Enterobacteriaceae (2.4\%). In the redundancy analyses (RDAs) (figure $2 \mathrm{~A}-\mathrm{C}$ ), the variation in the microbiota that could be explained by group at baseline was $0.5 \%(\mathrm{p}=0.048)$. There was a treatment signal at 3 weeks and 4 months: variation explained by the group at 3 weeks increased to $0.7 \%$ $(\mathrm{p}=0.014)$ (figure $2 \mathrm{~A})$ and was $0.8 \%$ at 4 months $(\mathrm{p}=0.034)$ (figure 2B). In the ratio RDA of baseline to 4 months, the three groups were separated (trend, $\mathrm{p}=0.084$; variation explained by treatment $0.7 \%$ ) (figure $2 \mathrm{C}$ ). 
Table 2 Age, gender, iron and inflammation variables, faecal calprotectin and faecal pH, by group. Differences in these variables among Kenyan infants $(n=155)$ at baseline and after 4 months of consuming daily a micronutrient powder containing either no iron (control), $5 \mathrm{mg}$ of iron (Fe), or $5 \mathrm{mg}$ of iron and $7.5 \mathrm{~g}$ GOS (FeGOS)

\begin{tabular}{|c|c|c|c|}
\hline & Control & $\mathrm{Fe}$ & FeGOS \\
\hline \multicolumn{4}{|l|}{ Age (month) } \\
\hline Baseline & $7.5(6.9,9.1)^{*}$ & $7.5(6.9,9.3)$ & $7.3(6.9,9.2)$ \\
\hline 4 months & $11.3(10.5,13.0)$ & $11.2(10.6,13.1)$ & $11.1(10.6,13.1)$ \\
\hline Male/Female & $28 / 23$ & $27 / 25$ & $22 / 30$ \\
\hline \multicolumn{4}{|c|}{ Haemoglobin (g/L) } \\
\hline Baseline & $103(97-110) \dagger$ & $103(93-108)$ & $106(102-111)$ \\
\hline 4 months & $101(93-108)^{a}$ & $112(107-122)^{b}$ & $111(103-116)^{b}$ \\
\hline \multicolumn{4}{|c|}{ Anaemia, n (\%) } \\
\hline Baseline & $38(75)$ & $41(79)$ & $36(69)$ \\
\hline 4 months & $40(83)^{\mathrm{a}}$ & $17(35)^{b}$ & $19(42)^{b}$ \\
\hline \multicolumn{4}{|c|}{ Plasma ferritin ( $\mu \mathrm{g} / \mathrm{L})$} \\
\hline Baseline & $13.6(8.2-28.5)$ & $12.6(8.9-26.1)$ & $16.9(12.6-31.3)$ \\
\hline 4 months & $12.4(8.4-26.3)^{\mathrm{a}}$ & $22.8(17.4-33.4)^{b}$ & $24.2(16.5-36.2)^{b}$ \\
\hline \multicolumn{4}{|c|}{ Soluble transferrin receptor (mg/L) } \\
\hline Baseline & $10.8(8.4-14.9)$ & $12.6(8.7-16.1)$ & $11.6(8.8-15.8)$ \\
\hline 4 months & $12.6(10.0-17.5)^{\mathrm{a}}$ & $9.6(8.0-11.5)^{b}$ & $8.4(7.7-10.2)^{b}$ \\
\hline \multicolumn{4}{|c|}{ Iron deficiency anaemia, $\mathrm{n}(\%)$} \\
\hline Baseline & $35(69)$ & $38(73)$ & $32(62)$ \\
\hline 4 months & $35(73)^{\mathrm{a}}$ & $13(27)^{b}$ & $18(40)^{b}$ \\
\hline \multicolumn{4}{|c|}{$\mathrm{C}$ reactive protein $(\mathrm{mg} / \mathrm{L})$} \\
\hline Baseline & $1.2(0.5-6.7)$ & $1.2(0.5-3.2)$ & $1.2(0.5-6.5)$ \\
\hline 4 months & $1.2(0.4-3.0)$ & $1.4(0.6-3.4)$ & $1.4(0.4-6.9)$ \\
\hline \multicolumn{4}{|c|}{ Alpha-glycoprotein (g/L) } \\
\hline Baseline & $0.9(0.7-1.6)$ & $1.0(0.7-1.6)$ & $1.0(0.7-1.8)$ \\
\hline 4 months & $1.1(0.7-1.9)$ & $1.2(0.7-1.8)$ & $1.0(0.7-1.9)$ \\
\hline \multicolumn{4}{|c|}{ Faecal calprotectin $(\mu \mathrm{g} / \mathrm{g})$} \\
\hline Baseline & $191.9(107.0-304.7)$ & $202.2(111.6-312.9)$ & $236.9(120.6-322.0)$ \\
\hline 3 weeks & $193.3(100.0-321.8)$ & $198.8(89.3-387.0)$ & $163.0(77.8-294.2)$ \\
\hline 4 months & $123.7(45.2-245.1)$ & $136.1(62.7-243.5)$ & $147.8(77.2-308.6)$ \\
\hline \multicolumn{4}{|l|}{ Faecal pH } \\
\hline Baseline & $5.7(5.2-6.2)$ & $5.5(5.0-6.1)$ & $5.6(5.2-6.1)$ \\
\hline 3 weeks & $5.5(5.1-5.9)^{\mathrm{a}}$ & $5.9(5.4-6.5)^{b}$ & $5.4(4.6-6.0)^{\mathrm{a}}$ \\
\hline 4 months & $5.6(5.3-6.2)$ & $5.6(5.3-6.0)$ & $5.6(5.3-6.0)$ \\
\hline
\end{tabular}

For continuous variables, between-group differences at baseline were tested using one-factor analysis of variance: there were no significant baseline differences between any of the variables. Between-group differences at study endpoint were tested using analysis of covariance with baseline values as covariates. For categorical variables, between-group differences at baseline and endpoint were tested using generalised linear models. Across rows, different letter superscripts indicate significant differences; $p<0.05$.

*Median (min, max), all such values.

tMedian (IQR), all such values.

GOS, galacto-oligosaccharides.

Maturation of the infant gut microbiome during the study was reflected in the phylogenetic distance from baseline to 3 weeks and from baseline to 4 months within each individual, as shown in the weighted UniFrac distances (figure 2D). The phylogenetic distance from 0 to 3 weeks and from 0 to 4 months among the intervention groups differed (trend, $p=0.079$ and $p=0.055$, respectively), and for $0-4$ months the phylogenetic distance in the Fe group was larger than in the FeGOS group (trend, $p=0.063$ ), while there was no significant difference in phylogenetic distance comparing the FeGOS and control groups. In the Fe group at 4 months, there was a shift towards a more mature, adult-like composition ${ }^{21} 22$ enriched in taxa such as Roseburia, Clostridium, Anaerostipes and Ruminococcus, while less mature, infant-associated taxa including Collinsella, Lactobacillus, Bifidobacterium and Megasphaera were associated with the control and FeGOS (see online supplementary figure 1 ).
Between-group differences in all taxa at 3 weeks and 4 months are shown in figures $3 \mathrm{~A}-\mathrm{C}$ and $4 \mathrm{~A}-\mathrm{C}$, respectively, and differences in taxa of our primary interest (Bifidobacterium, Lactobacillus, Enterobacteriaceae, Clostridiales and Bacteroidetes) at 3 weeks and 4 months are shown in the inset simplified figures $3 \mathrm{D}-\mathrm{F}$ and $4 \mathrm{D}-\mathrm{F}$, respectively. Fe treatment had a major impact on the microbiome composition compared with FeGOS and control at 3 weeks and 4 months. At 4 months, compared with the control group, in the Fe group (figure 4D), there were lower abundances of the genera Lactobacillus $(\mathrm{p}=0.048)$, lower abundances of the genera Bifidobacterium (trend, $\mathrm{p}=0.058$ ), higher abundances of the order Clostridiales $(\mathrm{p}=0.015)$ and higher abundances of the family Enterobacteriaceae (trend, $\mathrm{p}=0.086$ ), but no significant differences in phylum Bacteroidetes. At 4 months, compared with the FeGOS group, in the Fe group (figure 4E), there were lower abundances of the genera Bifidobacterium $(p=0.007)$ 

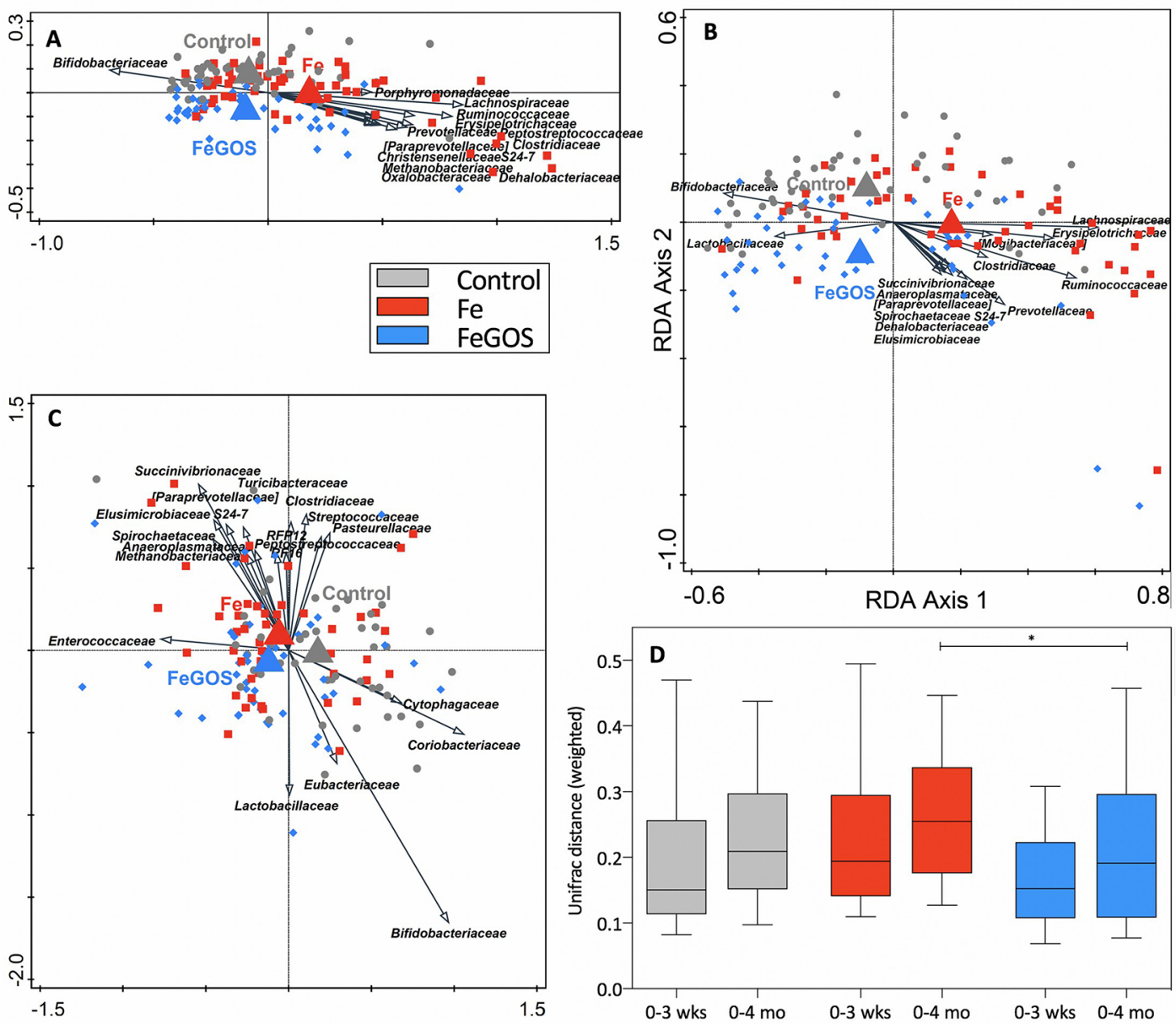

Figure 2 Redundancy analysis (RDA) and phylogenetic distances, by group. Kenyan infants $(n=155)$ receiving daily a micronutrient powder containing either no iron (control); $5 \mathrm{mg}$ of iron (Fe); or $5 \mathrm{mg}$ of iron and $7.5 \mathrm{~g}$ of galacto-oligosaccharides (FeGOS): in the RDAs, the variation explained by group was (A) $0.7 \%$ at 3 weeks $(p=0.014)$ and $(B) 0.8 \%$ at 4 months $(p=0.034)$; $(C)$ the ratio RDA of baseline to 4 months showed separation of the three groups (variation explained by treatment $0.7 \%$; trend, $p=0.084$ ). (D) The phylogenetic distance (UniFrac weighted index) from baseline to 3 weeks ( $0-3$ weeks) and from baseline to 4 months ( $0-4$ months) within each infant. The phylogenetic distance from 0 to 3 weeks and from 0 to 4 months among the intervention groups differed (trend, $p=0.079$ and $p=0.055$, respectively; Kruskal-Wallis test), and for $0-4$ months was larger in Fe compared with FeGOS (trend, * $p=0.063$; Dunn's post-hoc test).

and Lactobacillus $(\mathrm{p}=0.006)$ and higher abundances of the order Clostridiales $(\mathrm{p}=0.001)$, but no significant difference in the abundance of Enterobacteriaceae and Bacteroidetes. In addition, at 4 months, Ruminococcaceae and Lachnospiraceae (mainly Dorea, Blautia and Coprococcus) and Erysipelotrichaceae were significantly more abundant in the Fe group compared with FeGOS and control (all, p<0.05). Remarkably, comparing the control and FeGOS groups, only a few low abundance taxa differed, and there were no significant differences in the abundances of Bifidobacterium, Lactobacillus, Enterobacteriaceae, Clostridiales and Bacteroidetes (figure 4F).

\section{Occurrence and abundance of gut pathogens by qPCR}

From all analysed stool samples at baseline $(n=155)$, we detected C. perfringens in 65\% (median in detected samples: 4.6 log gene copies/g faeces (IQR: 4.1-5.5)), C. difficile in 35\% (5.6 (4.6-6.0)), S. aureus in $13 \%(4.7(4.5-5.2))$, B. cereus in 5\% (4.7 (4.4-5.5)) and Salmonella spp in 4\% (4.5 (4.3-5.2)). Further, we detected EPEC in 63\% (5.5 (4.8-6.5)), the heat-labile enterotoxin (ETEC $L T)$ and heat-stable enterotoxin (ETEC ST) of enterotoxigenic E. coli in $26 \%(6.6(6.1-8.0))$ and $21 \%(4.4(3.8-5.7))$, respectively, and Shiga toxin 2 (EHEC stx2) and Shiga toxin 1 (EHEC stx1) of enterohemorrhagic E. coli in $18 \%(4.3(4.0-4.8))$ and $8 \%(4.3$ (3.94.9)), respectively. There were no significant differences among groups at baseline in any of these VTGs. At 4 months, in samples positive for EHEC stx2, there were lower abundances in the FeGOS group (4.0 (3.6-4.5)) compared with the Fe (4.8 (4.3-5.3)) and control groups $(4.9(4.7-5.3))(p=0.036$ and $p=0.012$, respectively). For the sum of the 10 VTGs of all pathogens, at 3 weeks there were lower abundances in the FeGOS group (median (IQR), 5.7 (4.9-6.5)) compared with both the control group (6.3 (5.6$7.1))$ and the Fe group $(6.4(5.7-6.9))(p=0.005$ and $p=0.007$, respectively) (figure $5 \mathrm{~A}$ ). For the sum of VTGs of the pathogenic E. coli, at 3 weeks, there were lower abundances in the FeGOS group (5.8 (4.9-6.4)) compared with the control group (6.3 (5.5$7.1))(p=0.035)$. At 4 months there were no significant differences in the VTGs of all pathogens or in the VTGs of pathogenic E. coli among groups. Within groups, from baseline to 4 months, there was a decrease in the VTGs of all pathogens in the FeGOS group $(5.9(5.3-6.7)$ to $5.4(4.6-6.3))(\mathrm{p}=0.024)$ and in the control group $(6.0(5.2-6.7)$ to $5.4(4.1-6.4))(\mathrm{p}=0.037)$, but no significant change in the Fe group. Correlations between enteropathogens 

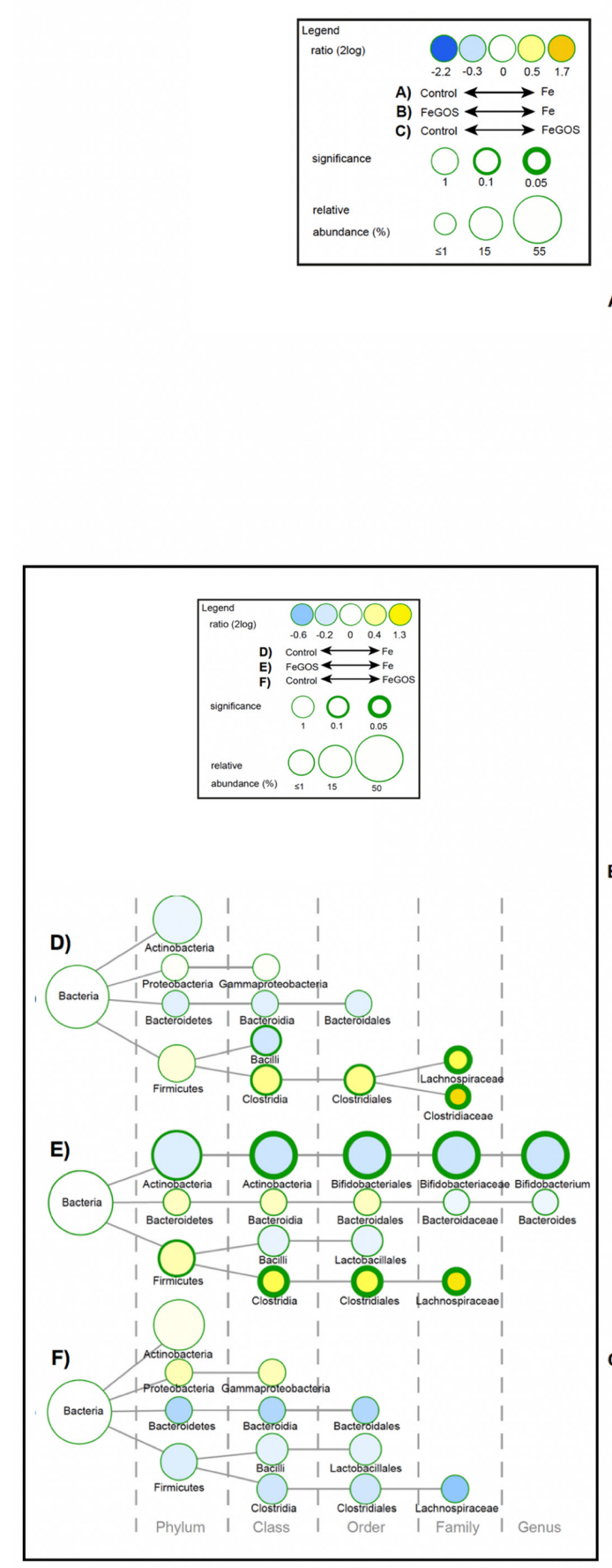

A)

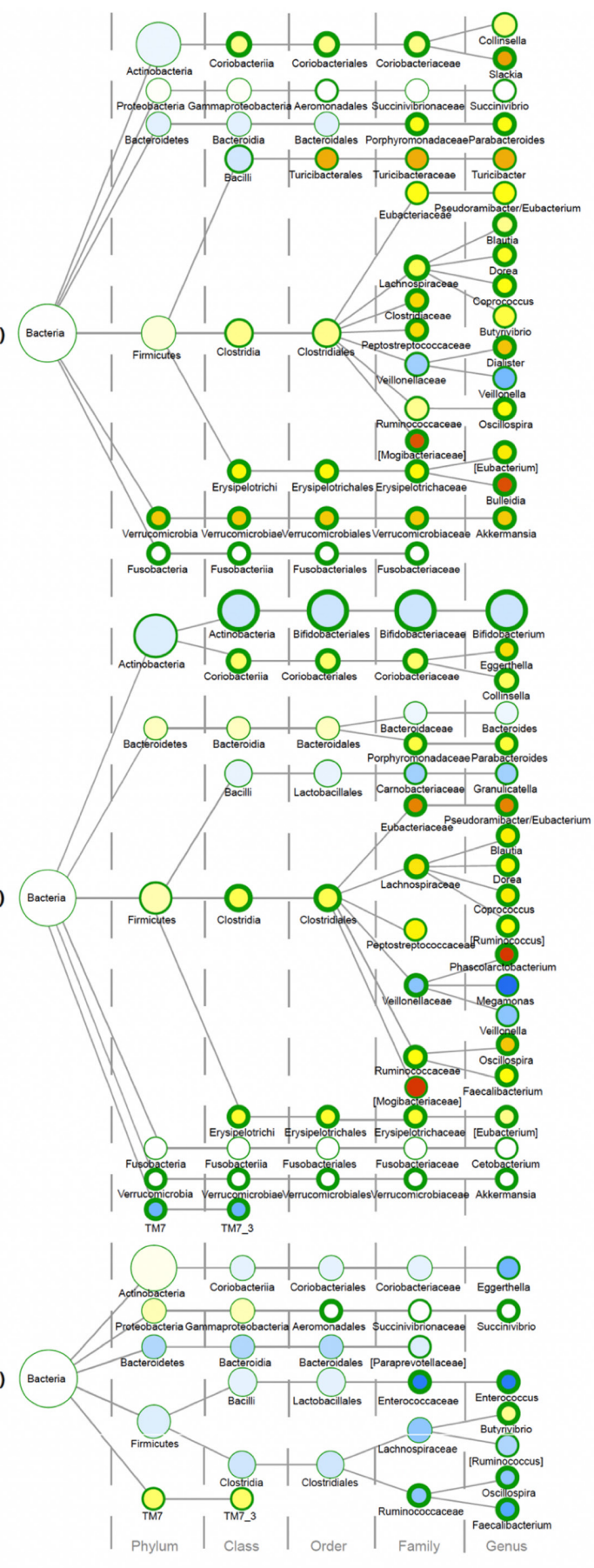

Figure 3 Group differences in gut microbial composition at 3 weeks. Differences in gut microbial composition in Kenyan infants $(n=155)$ after 3 weeks of receiving daily a micronutrient powder containing either no iron (control); $5 \mathrm{mg}$ of iron (Fe); or $5 \mathrm{mg}$ of iron and $7.5 \mathrm{~g}$ of galactooligosaccharides (FeGOS): (A-C) All taxa. (D-F) Inset box shows only taxa that were prespecified targets of interest. Nodes represent taxa; edges link the different taxonomic levels. The node-size corresponds to the relative abundance (in \%). The fold difference is calculated as the $2 \log$ of the ratio of the relative abundance between groups. Significance is expressed as the $p$ value of a Mann-Whitney $\mathrm{U}$ test. 

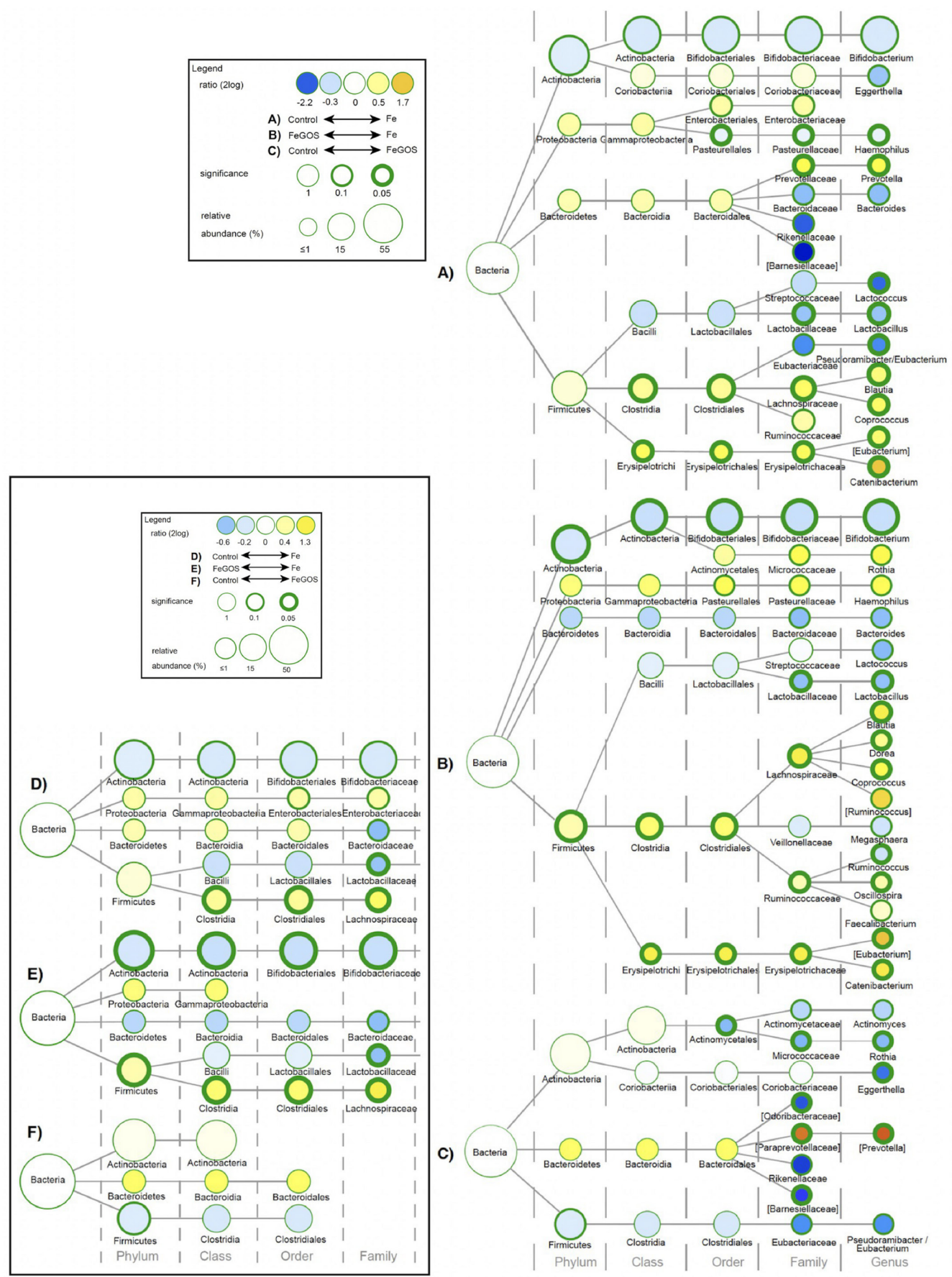

Figure 4 Group differences in gut microbial composition at 4 months. Differences in gut microbial composition in Kenyan infants $(n=155)$ after 4 months of receiving daily a micronutrient powder containing either no iron (control); $5 \mathrm{mg}$ of iron (Fe); or $5 \mathrm{mg}$ of iron and $7.5 \mathrm{~g}$ of galactooligosaccharides (FeGOS). (A-C) All taxa. (D-F) Inset box shows only taxa that were prespecified targets of interest. Nodes represent taxa; edges link the different taxonomic levels. The node-size corresponds to the relative abundance (in \%). The fold difference is calculated as the $2 \log$ of the ratio of the relative abundance between groups. Significance is expressed as the $p$ value of a Mann-Whitney $\mathrm{U}$ test; exact $p$ values for significance are in the text. 


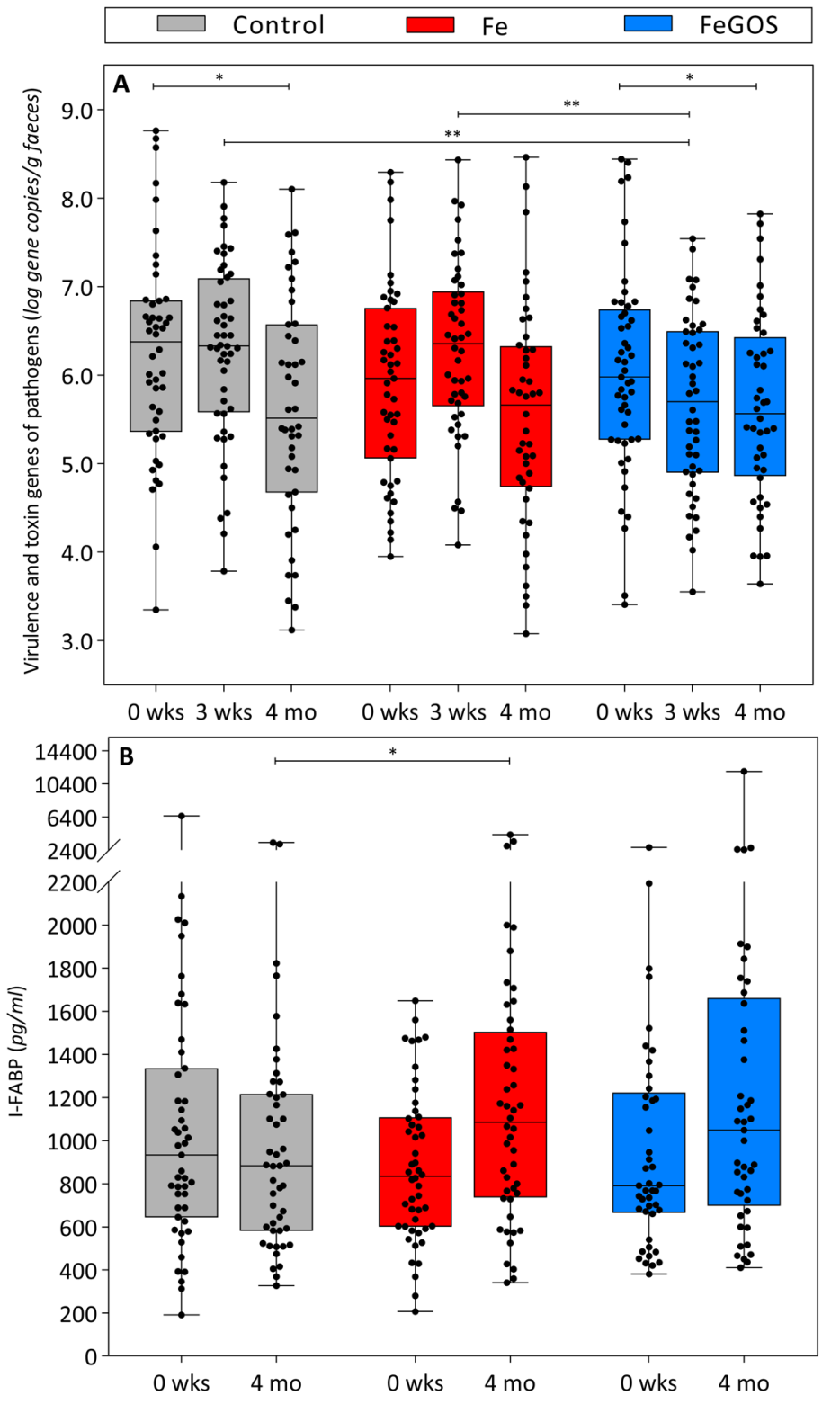

Figure 5 Abundances of the sum of virulence and toxin genes of the 10 targeted pathogens and intestinal fatty acid-binding protein (I-FABP), by group. Kenyan infants $(n=155)$ receiving daily a micronutrient powder containing either no iron (control); containing 5 mg of iron (Fe); or containing $5 \mathrm{mg}$ of iron and $7.5 \mathrm{~g}$ of galacto-oligosaccharides (FeGOS). (A) The abundances of the sum of virulence and toxin genes of the 10 targeted pathogens (online supplementary table 1) at baseline, 3 weeks and 4 months, by group, ${ }^{*} p<0.05,{ }^{* *} p<0.01$. Significance expressed as the $p$ value of a Wilcoxon rank-sum test. (B) I-FABP at baseline and 4 months, by group, ${ }^{*} p=0.0498$. Significance expressed as the $p$ value of analysis of covariance with baseline values as covariate. Boxes show the median and 25th and 75th percentiles; whiskers show the range; points show individual values.

and markers of gut and systemic inflammation are given in the online supplementary material.

\section{Faecal calprotectin and $\mathrm{pH}$, and plasma I-FABP}

At 3 weeks and 4 months, there were no significant group differences in faecal calprotectin, although there was a non-significant $\approx 30 \%$ decrease from baseline to 3 weeks in the FeGOS group (table 2). At 3 weeks, faecal $\mathrm{pH}$ was higher in the Fe group than in the control and FeGOS groups $(\mathrm{p}=0.005$ and $\mathrm{p}=0.001$, respectively) (table 2). At 4 months, compared with the control group, I-FABP was higher in the Fe group $(p=0.0498)$ (figure $5 B)$.

\section{Infant morbidity}

For morbidity assessed at the weekly visits, there were no significant time by treatment effects on diarrhoea or fever, but there was a significant time by treatment effect on the incidence of RTIs, with a decrease in RTIs over time in the FeGOS group $(p=0.039)$. During the 4-month intervention, we recorded 465 treated cases in the health clinics: 237 for RTI, 74 for diarrhoea, 56 for malaria, 39 for skin infection and 59 for other health problems. There were no significant group differences in the number of infants treated for diarrhoea or other health problems during the intervention. However, more infants in the 


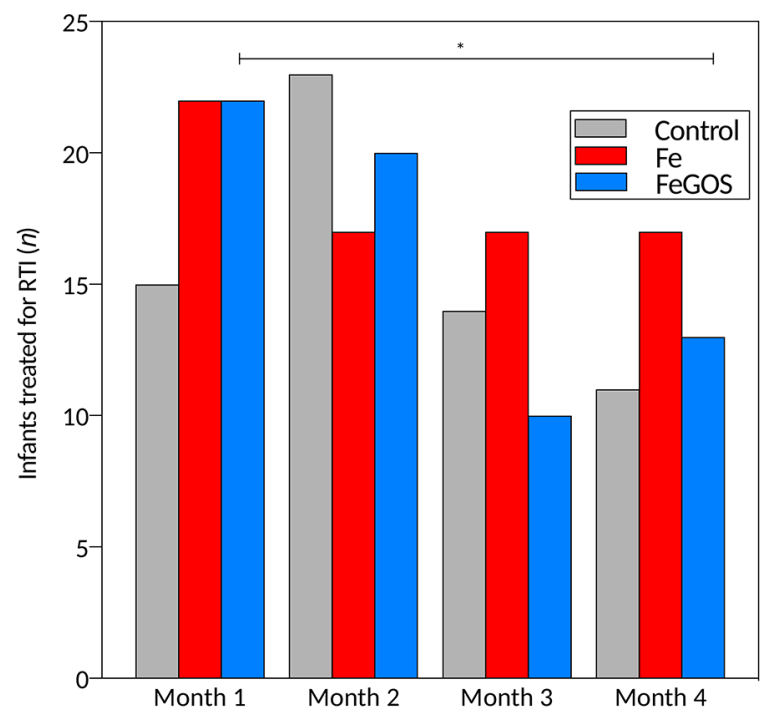

Figure 6 Treated respiratory tract infections (RTIs), by group. Kenyan infants ( $n=155)$ receiving daily a micronutrient powder containing either no iron (control); containing $5 \mathrm{mg}$ of iron (Fe); or containing $5 \mathrm{mg}$ of iron and $7.5 \mathrm{~g}$ of galacto-oligosaccharides (FeGOS). ${ }^{*} \mathrm{p}=0.013$. $p$ value expresses the significant time effect of a generalised linear model with infants treated for RTI as response variable, time as fixed factor and subject as a random effect.

Fe group were treated for a RTI during the intervention $(n=45$; $87 \%$ of all infants) compared with the control group $(n=38$; $75 \%)(p=0.024)$ and compared with the FeGOS group $(n=39$; $75 \%$ ) (trend, $\mathrm{p}=0.098$ ). Over the 4 months of the study, there was a decrease in treated cases of RTIs in the FeGOS group $(p=0.013)$ (figure 6) but no significant change in the other two groups.

\section{DISCUSSION}

Due to the potential adverse effects of unabsorbed iron on the infant gut, ${ }^{15}$ the iron dose in MNPs should be as low as possible while retaining efficacy against anaemia. In this study, daily use for 4 months of a MNP containing $5 \mathrm{mg}$ iron as ferrous fumarate and NaFeEDTA was clearly efficacious in reducing anaemia and all measures of iron deficiency (table 2). The haematological response comparing Fe and FeGOS was comparable, suggesting the addition of the GOS did not affect iron bioavailability. This $5 \mathrm{mg}$ dose is $60 \%$ lower than the $12.5 \mathrm{mg}$ in currently used MNPs, yet the improvements in $\mathrm{Hb}(\approx+5-9 \mathrm{~g} / \mathrm{L})$ and reductions in anaemia ( $\approx-30 \%-50 \%)$ compare favourably with those reported for $12.5 \mathrm{mg}$ iron MNPs. ${ }^{3}$

Previous intervention studies of the effect of iron on the infant and child microbiome are limited. ${ }^{23}$ In a controlled trial in 6-month-old Kenyan infants, iron-containing MNPs increased the Enterobacteriaceae:Bifidobacteriaceae ratio and numbers of enteropathogenic E. coli. ${ }^{15}$ In contrast, in 6-month-old Malawian infants randomised to no intervention or dietary supplements providing 5.5-6.0 mg iron/day, there were no significant differences in the gut microbiota after 12 months. $^{24}$ In a controlled trial in Ivorian schoolaged children receiving iron-fortified biscuits $(\approx 9 \mathrm{mg}$ iron/ day), iron increased Enterobacteriaceae and decreased Lactobacillaceae. ${ }^{19}$ In contrast, in South African school-aged children from an area with better hygiene, iron supplements
(50 mg/day, 4 days/week) caused no discernible effects on the gut microbiota. ${ }^{25}$ The varied findings from these studies are likely due to age-related differences in the gut microbiota between infants and older children, differences in methods used to characterise the gut microbiome, differences in geographical setting and hygiene and/or the iron compound and dose given.

In our study, addition of GOS to an iron-containing MNP counterbalanced the effects of iron: after 4 months of the intervention in the Fe group, there were lower relative abundances of Bifidobacteriaceae and Lactobacillaceae, and higher relative abundances of Clostridiales, compared with the control and FeGOS groups; in contrast, there were no significant differences in these taxa comparing the FeGOS group with the control group (Figure 4F). We chose a GOS dose of $7.5 \mathrm{~g} /$ day based on studies that reported a bifidogenic effect in infants and adults at this dose and because this is the typical daily dose delivered by GOS-containing commercial infant formulas. ${ }^{12} 17$ The enhancement of commensal bacteria strengthens the barrier effect against enteropathogen growth: commensal bacteria occupy more microbiological niches, compete for nutrients, increase secretory IgA and reduce pathogen adhesion site. ${ }^{12} 17$ The suppressive effect on enteropathogens was visible in our data: during the intervention, the FeGOS group had lower abundances of the VTGs of all pathogens (figure 5A) and the VTGs of pathogenic E. coli compared with the Fe and control groups. Prebiotics also increase commensal production of SCFAs that decrease luminal $\mathrm{pH}$, which may reduce growth of pathogens, ${ }^{26}{ }^{27}$ and in our study, at 3 weeks, faecal $\mathrm{pH}$ was lower in the FeGOS group than in the Fe group (table 2).

Previous studies in African infants ${ }^{15}$ and children ${ }^{19}$ have shown that iron fortification increases faecal calprotectin. Faecal calprotectin is a non-specific marker of gut inflammation that reflects neutrophil infiltration of the mucosa ${ }^{28} 29$; levels are normally high in early infancy and decrease over the first 2 years as the gut matures. ${ }^{15}$ In contrast to our previous MNP trial in Kenyan infants where a $12.5 \mathrm{mg}$ iron dose increased faecal calprotectin, the $5 \mathrm{mg}$ dose used here (and a $2.5 \mathrm{mg}$ dose used previously ${ }^{15}$ ) did not increase faecal calprotectin (table 2), suggesting that daily doses of $\leq 5 \mathrm{mg}$ iron may not trigger gut inflammation in this setting. Damaged enterocytes release I-FABP into the bloodstream ${ }^{30}$; it is a sensitive marker for enterocyte injury and can be combined with faecal calprotectin to assess disease severity. ${ }^{31}$ I-FABP in our infants at baseline was higher than in European children with sepsis, ${ }^{32}$ and high plasma I-FABP in African infants may reflect enterocyte injury due to common enteric infections. ${ }^{33}$ In our study, at 4 months, I-FABP was higher in the Fe group than in the control group, while there was no significant difference comparing the FeGOS group with control (figure 5B), suggesting the addition of GOS may have reduced Fe-mediated enterocyte damage; this effect may be mediated through changes in gut microbiota ${ }^{34}$ and/or iron-induced oxidative stress to enterocytes. ${ }^{35}$

Compared with the Fe group, there was a lower incidence of RTIs in the FeGOS group and a decrease in RTIs in the FeGOS group over the 4-month intervention (figure 6), but no group differences in diarrhoea. In our previous MNP study in Kenyan infants, there was a non-significant increase in incidence of diarrhoea with the $12.5 \mathrm{mg}$ Fe dose, but no increase in RTIs. ${ }^{15}$ This difference might be explained by the lower dose of iron in the present study, and/or because the infants in the present study were $\approx 2$ months older at entry. In a large MNP study (12.5 mg iron/day) that systematically collected morbidity data in Pakistani infants aged 6-18 months, there was an increased 
incidence of RTIs and diarrhoea with iron. ${ }^{7}$ Controlled trials in industrialised countries have reported that GOS alone or in combination with other prebiotics and probiotics reduced RTIs in infants. ${ }^{3637}$ Possible mechanisms of how prebiotics and probiotics reduce RTIs include reduced pathogen colonisation in the respiratory epithelium, ${ }^{38}$ regulation of mucosal immunity through activation of inflammasomes and modulation of the systemic immune response. ${ }^{39}$

Strengths of our study include (1) its double-blind, randomised controlled design; (2) a study population with high carriage rates of enteropathogens; (3) low attrition rate $(\approx 6 \%)$ during the study; and (4) extensive characterisation of the gut microbiome using the complementary methods of sequencing and qPCR for selected target species. We used maltodextrin in the control and Fe groups as a neutral comparator to the GOS because glucose from maltodextrin is rapidly absorbed in the proximal small intestine, ${ }^{40}$ so its presence is unlikely to have influenced our comparisons. A limitation of our study is that despite randomisation, there were small baseline differences in gut microbiota composition among the groups, which may have affected our comparisons. Also, because of the small sample size and high between-subject variability for the gut microbiota, a beta error may have contributed to non-significant comparisons. In addition, we characterised the gut microbiome in faecal samples, which may not necessarily represent bacterial abundances in the distal small intestine, caecum and proximal colon. ${ }^{40}$

In conclusion, a MNP containing a $5 \mathrm{mg}$ daily dose of highly bioavailable iron is efficacious in reducing IDA in African infants, and this low dose is likely safer in that it induces less adverse changes in the gut microbiome and no increase in faecal calprotectin compared with a $12.5 \mathrm{mg}$ iron dose. ${ }^{15}$ Our findings suggest that prebiotics given with iron-containing MNPs in the African setting may be beneficial to reduce the adverse effects of iron on the infant gut microbiome. Expert groups have judged prebiotics to be safe and well tolerated in infants ${ }^{18}$; however, nearly all previous studies were done in infants from high-income countries with good hygiene. Further studies confirming the safety and tolerability of GOS in the African setting are needed, as well as the smallest effective dose of GOS needed to offset the adverse effects of iron on the infant gut. In addition, studies combining GOS with iron fortificants or supplements in other geographical settings (eg, South Asia) and in older children would be valuable.

Acknowledgements We thank the families who participated in this study, the field workers and nurses from Msambweni County Referral Hospital (Msambweni, Kenya) and Kikoneni Health Centre (Kikoneni, Kenya), as well as C Zeder, A Krzystek, V Galetti, A Die, A Minder and S Kobel (ETH Zurich, Switzerland) and J Erhardt (Willstaett, Germany) for support of the laboratory and data analysis. This study is registered at ClinicalTrials.gov as NCT02118402.

Contributors DP, CIC, DM, TB-J, CL and MBZ designed the study; DP, MAU and $\mathrm{CIC}$ conducted the study; DP, GAMK, JB, HMT and MBZ analysed the data and performed the statistical analyses; DP, MAU, CIC, GAMK, DM, TB-J, CS, JB, HMT, CL, SK and MBZ participated in the data interpretation; DP, GAMK, CS and MBZ wrote the manuscript. All authors edited the manuscript. All authors read and approved the final version of the manuscript.

Funding ETH Global and the Sawiris Foundation for Social Development, ETH Zurich, Switzerland; DSM Nutritional Products, Kaiseraugst, Switzerland. sight and Life (Kaiseraugst, Switzerland) donated the MNPs used in this study. FrieslandCampina (Wageningen, The Netherlands) donated the GOS used in this study.

Ethics approval Kenyatta National Hospital/University of Nairobi, Kenya (P521/10/2013) and the Zurich Cantonal Ethical Commission (2014-0232).

Provenance and peer review Not commissioned; externally peer reviewed.
(C) Article author(s) (or their employer(s) unless otherwise stated in the text of the article) 2017. All rights reserved. No commercial use is permitted unless otherwise expressly granted.

\section{REFERENCES}

1 WHO. The global prevalence of anaemia in 2011. Geneva: World Health Organization 2015.

2 Kassebaum NJ, Jasrasaria R, Naghavi M, et al. A systematic analysis of global Anemia burden from 1990 to 2010. Blood 2014;123:615-24.

3 De-Regil LM, Suchdev PS, Vist GE, et al. Home fortification of foods with multiple micronutrient powders for health and nutrition in children under two years of age (Review). Evid Based Child Health 2013;8:112-201.

4 Neuberger A, Okebe J, Yahav D, et al. Oral iron supplements for children in malariaendemic areas. Cochrane Database Syst Rev 2016;2:CD006589.

5 Salam RA, MacPhail C, Das JK, et al. Effectiveness of Micronutrient Powders (MNP) in women and children. BMC Public Health 2013;13(Suppl 3):S22.

6 Zlotkin S, Newton S, Aimone AM, et al. Effect of iron fortification on malaria incidence in infants and young children in Ghana: a randomized trial. JAMA 2013;310:938-47.

7 Soofi S, Cousens S, Iqbal SP, et al. Effect of provision of daily zinc and iron with several micronutrients on growth and morbidity among young children in Pakistan: a clusterrandomised trial. Lancet 2013;382:29-40.

8 Tondeur MC, Schauer CS, Christofides AL, et al. Determination of iron absorption from intrinsically labeled microencapsulated ferrous fumarate (sprinkles) in infants with different iron and hematologic status by using a dual-stable-isotope method. Am J Clin Nutr 2004;80:1436-44.

9 Andrews SC, Robinson AK, Rodríguez-Quiñones F. Bacterial iron homeostasis. FEMS Microbiol Rev 2003;27:215-37.

10 Kortman GA, Raffatellu M, Swinkels DW, et al. Nutritional iron turned inside out: intestinal stress from a gut microbial perspective. FEMS Microbiol Rev 2014:38:1202-34.

11 Fukuda S, Toh H, Hase K, et al. Bifidobacteria can protect from enteropathogenic infection through production of acetate. Nature 2011;469:543-7.

12 Bertelsen RJ, Jensen ET, Ringel-Kulka T. Use of probiotics and prebiotics in infant feeding. Best Pract Res Clin Gastroenterol 2016:30:39-48.

13 Weinberg ED. The Lactobacillus anomaly: total iron abstinence. Perspect Biol Med 1997;40:578-83.

14 Vazquez-Gutierrez P, Lacroix C, Jaeggi T, et al. Bifidobacteria strains isolated from stools of iron deficient infants can efficiently sequester iron. BMC Microbiol 2015;15:3.

15 Jaeggi T, Kortman GA, Moretti D, et al. Iron fortification adversely affects the gut microbiome, increases pathogen abundance and induces intestinal inflammation in Kenyan infants. Gut 2015;64:731-42.

16 Troesch B, Egli I, Zeder C, et al. Optimization of a phytase-containing micronutrient powder with low amounts of highly bioavailable iron for in-home fortification of complementary foods. Am J Clin Nutr 2009;89:539-44.

17 Roberfroid M, Gibson GR, Hoyles L, et al. Prebiotic effects: metabolic and health benefits. Br J Nutr 2010;104-(Suppl 2)-S1-S63.

18 FDA. GRAS Notification (GRN No. 236) for Galacto-oligosaccharides (GOS), 2007.

19 Zimmermann MB, Chassard C, Rohner F, et al. The effects of iron fortification on the gut microbiota in African children: a randomized controlled trial in Cote d'Ivoire. Am J Clin Nutr 2010;92:1406-15.

20 Erhardt JG, Estes JE, Pfeiffer CM, et al. Combined measurement of ferritin, soluble transferrin receptor, retinol binding protein, and C-reactive protein by an inexpensive, sensitive, and simple sandwich enzyme-linked immunosorbent assay technique. J Nutr 2004; 134:3127-32.

21 Bäckhed F, Roswall J, Peng Y, et al. Dynamics and Stabilization of the Human Gut Microbiome during the First Year of Life. Cell Host Microbe 2015;17:852.

22 Subramanian S, Huq S, Yatsunenko T, et al. Persistent gut microbiota immaturity in malnourished Bangladeshi children. Nature 2014;510:417-21.

23 Paganini D, Uyoga M, Zimmermann M. Iron Fortification of Foods for Infants and Children in Low-Income Countries: Effects on the Gut Microbiome, Gut Inflammation, and Diarrhea. Nutrients 2016;8:494.

24 Cheung YB, Xu Y, Mangani C, et al. Gut microbiota in Malawian infants in a nutritional supplementation trial. Trop Med Int Health 2016;21:283-90.

25 Dostal A, Baumgartner J, Riesen N, et al. Effects of iron supplementation on dominant bacterial groups in the gut, faecal SCFA and gut inflammation: a randomised, placebo-controlled intervention trial in South African children. Br J Nutr 2014;112:547-56.

26 Ríos-Covián D, Ruas-Madiedo P, Margolles A, et al. Intestinal Short Chain Fatty Acids and their link with Diet and Human Health. Front Microbiol 2016;7.

27 Rivera-Chávez F, Zhang LF, Faber F, et al. Depletion of Butyrate-Producing Clostridia from the Gut Microbiota Drives an Aerobic Luminal Expansion of Salmonella. Cell Host Microbe 2016;19:443-54.

28 Berni Canani R, Rapacciuolo L, Romano MT, et al. Diagnostic value of faecal calprotectin in paediatric gastroenterology clinical practice. Dig Liver Dis 2004;36:467-70. 
29 Sýkora J, Siala K, Huml M, et al. Evaluation of faecal calprotectin as a valuable non-invasive marker in distinguishing gut pathogens in young children with acute gastroenteritis. Acta Paediatr 2010;99:1389-95.

30 Pelsers MM, Namiot Z, Kisielewski W, et al. Intestinal-type and liver-type fatty acidbinding protein in the intestine. Tissue distribution and clinical utility. Clin Biochem 2003:36:529-35.

31 Reisinger KW, Van der Zee DC, Brouwers HA, et al. Noninvasive measurement of fecal calprotectin and serum amyloid A combined with intestinal fatty acid-binding protein in necrotizing enterocolitis. J Pediatr Surg 2012;47:1640-5.

32 Derikx JP, Bijker EM, Vos GD, et al. Gut mucosal cell damage in meningococcal sepsis in children: relation with clinical outcome. Crit Care Med 2010;38:133-7.

33 Prendergast AJ, Rukobo S, Chasekwa B, et al. Stunting is characterized by chronic inflammation in Zimbabwean infants. PLoS One 2014;9:e86928.

34 Gonçalves FL, Soares LM, Figueira RL, et al. Evaluation of the expression of I-FABP and L-FABP in a necrotizing enterocolitis model after the use of Lactobacillus acidophilus. J Pediatr Surg 2015;50:543-9.

35 Carrier J, Aghdassi E, Cullen J, et al. Iron supplementation increases disease activity and vitamin $\mathrm{E}$ ameliorates the effect in rats with dextran sulfate sodium-induced colitis. J Nutr 2002;132:3146-50.
36 Arslanoglu S, Moro GE, Boehm G. Early supplementation of prebiotic oligosaccharides protects formula-fed infants against infections during the first 6 months of life. J Nutr 2007; 137:2420-4.

37 Luoto $\mathrm{R}$, Ruuskanen $\mathrm{O}$, Waris $\mathrm{M}$, et al. Prebiotic and probiotic supplementation prevents rhinovirus infections in preterm infants: a randomized, placebo-controlled trial. J Allergy Clin Immunol 2014;133:405-13.

38 Glück U, Gebbers JO. Ingested probiotics reduce nasal colonization with pathogenic bacteria (Staphylococcus aureus, Streptococcus pneumoniae, and beta-hemolytic streptococci). Am J Clin Nutr 2003;77:517-20.

39 Ichinohe T, Pang IK, Kumamoto Y, et al. Microbiota regulates immune defense against respiratory tract influenza A virus infection. Proc Natl Acad Sci U S A 2011;108:5354-9.

40 Hofman DL, van Buul VJ, Brouns FJ. Nutrition, Health, and Regulatory Aspects of Digestible Maltodextrins. Crit Rev Food Sci Nutr 2016;56:2091-100.

41 Gerritsen J, Smidt H, Rijkers GT, et al. Intestinal microbiota in human health and disease: the impact of probiotics. Genes Nutr 2011;6:209-40. 